\title{
Chemical Modification of Urokinase with Human Serum Albumin Fragments
}

\author{
Kumio Yokorgawa, * Katsuyuki Tanizawa** and Kenji Soda*** \\ Laboratory of Microbial Biochemistry, Institute for Chemical Research, \\ Kyoto University, Uji, Kyoto 611, Japan \\ Received April 19, 1989
}

\begin{abstract}
A therapeutically important fibrinolytic enzyme, urokinase, has been modified with fragmented human serum albumin (mol wt., $1000 \sim 7000$ ) by reaction with glutaraldehyde as a coupling agent. The chemically modified enzyme was separated by gel filtration with Sephadex G-150 into products which underwent conjugation with albumin fragments to various extents. The major modification product, with a molecular weight of about $\mathbf{7 0 , 0 0 0}$, and retaining $\mathbf{4 0} \%$ of the original urokinase activity, was significantly more resistant to inactivation by protease inhibitors in rat plasma than the unmodified enzyme. It also showed an in vivo half-life ten times longer than the native one during circulation in blood. The rabbit antiserum raised against the modified urokinase reacted not only with the antigen but also with both the unmodified enzyme and the human serum albumin. The modified enzyme did not elicit specific antibodies in an immunized rabbit, and the enzyme that had been modified excessively with albumin fragments lost the antigenicity of urokinase. These results indicate that the chemical modification with albumin fragments is useful for improvement of in vitro and in vivo stabilities of chemotherapeutic enzymes.
\end{abstract}

A large variety of enzymes have been used clinically as chemotherapeutic agents. However, various side effects such as anaphylaxis and immunoreactions are exerted, usually by administration of foreign proteins. In addition, the administered enzymes are degraded rapidly by clearing systems during circulation in blood before they show their therapeutic effects. In an attempt to mask these undesirable properties of therapeutic enzymes, chemical modifications with natural and synthetic polymers including human serum albumin (HSA), ${ }^{1)}$ dextran, ${ }^{2)}$ and polyethylene glycol $(\mathrm{PEG})^{2 \sim 4)}$ have been used for several enzymes. However, albumin is a protein with a large molecular size, producing steric hindrance upon its attachment, and therefore is unsuitable for modification of enzymes acting on large molecules. ${ }^{1)}$ Dextran has been shown to induce higher immunogenicity of the modified enzyme than the unmodified one, ${ }^{2)}$ and PEG, which is an essentially inert polymer, also has been reported to enhance the clotting rate of fibrinogen solution by thrombin, ${ }^{5)}$ and hence is inappropriate to modification of fibrinolytic enzymes. Thus, the polymers used for chemical modification of therapeutic enzymes need to be small, non-immunogenic, and biologically inert.

Urokinase (UK, EC 3.4.21.31) is a fibrinolytic enzyme which is excreted into urine and catalyzes the conversion of plasminogen into the active form, plasmin. ${ }^{6,7)}$ Although UK is effective for the treatment of acute thromboembolic symptoms, ${ }^{8)}$ the injected UK is degraded very rapidly by the clearance system in the liver ${ }^{9}$; its half-life in blood is less than a minute. To prepare a clinically favorable UK

* On study leave from the Research Department, Wakamoto Pharmaceutical Co., Ashigarakami-gun, Kanagawa 258, Japan.

** Present address: Institute of Scientific and Industrial Research, Osaka University, Mohogaoka 8-1, Ibaraki, Osaka 567, Japan.

*** To whom all correspondence should be addressed. 
with a longer in vivo life but without newly gained specific immunogenicity, we modified it chemically with fragmented HSA. We here report markedly increased in vivo stability of the modified UK, and demonstrate the usefulness of HSA fragments for chemical modification of therapeutic enzymes.

\section{Materials and Methods}

Materials. UK was purified to homogeneity from human urine as described previously. ${ }^{10}$ HSA and glutaraldehyde ( $25 \%$ solution) were purchased from Wako Pure Chemical Industries, Osaka; bovine fibrinogen containing plasminogen was from Poviet Organon-Teknika, the Netherlands; thrombin was from Mochida Pharmaceutical, Tokyo; Freund's complete adjuvant was from Difco, Detroit, Mich.; bovine pancreas trypsin immobilized on beaded agarose ( $N$-tosyl-L-phenylalanine chloromethylketone-treated, $75 \sim 100$ units $/ \mathrm{ml}$ of gel) was from Sigma, St. Louis, Mo.; and sodium pentobarbital was from Pitman-Moore, Washington Crossing, N. J. All other chemicals were analytical grade reagents.

Enzyme and protein assays. The fibrinolytic activity of UK was measured on fibrin gel plates containing plasminogen, ${ }^{11)}$ and expressed as International Units (IU) ${ }^{12)}$ with the reference UK (MM-005) which was supplied by the National Institute of Hygienic Sciences, Tokyo. The specific activity was expressed as IU per mg protein. Protein was measured by the method of Lowry et al. ${ }^{13}$ ) with bovine serum albumin as a standard.

Fragmentation of HSA. HSA was cleaved into peptide fragments with cyanogen bromide ${ }^{14)}$ and then with immobilized trypsin. ${ }^{15)}$ Briefly, HSA ( $1 \mathrm{~g}$ ) was dissolved in $20 \mathrm{ml}$ of $80 \%$ formic acid and treated with cyanogen bromide $(1 \mathrm{~g})$ at $4^{\circ} \mathrm{C}$ for $24 \mathrm{hr}$. The reaction was stopped by dialysis against $0.1 \mathrm{M}$ potassium phosphate buffer ( $\mathrm{pH} 7.4$ ) containing $0.01 \mathrm{M} \mathrm{CaCl}_{2}$. The immobilized trypsin $(25 \mathrm{ml}$, $75 \sim 100$ units of trypsin/ $/ \mathrm{ml}$ of beaded agarose) was suspended in the dialyzed solution (15 mg of the cyanogen bromide-treated $\mathrm{HSA} / \mathrm{ml}$, about $65 \mathrm{ml}$ ) and incubated at $37^{\circ} \mathrm{C}$ for $4 \mathrm{hr}$ with gentle stirring. The agarose gels were removed by filtration to stop the digestion. The solution obtained was filtered through an Amicon PM-10 membrane for removal of peptides with molecular weights higher than 10,000 . The HSA fragments thus obtained in the filtrate were analyzed by urea/sodium dodecyl sulfate (SDS)/polyacrylamide gel electrophoresis with myoglobin and its fragmented polypeptides as standards for molecular weight measurement. ${ }^{16)}$

Modification of UK with HSA fragments. UK $(2 \mathrm{mg})$ and the HSA fragments $(20 \mathrm{mg})$ were dissolved in $6 \mathrm{ml}$ of $0.1 \mathrm{M}$ phosphate buffer ( $\mathrm{pH} 7.4$ ) containing $4.8 \mathrm{mg}$ (19.2 $\mu$ l of $25 \%$ solution) of glutaraldehyde. After the coupling reaction at $4 \mathrm{C}$ for $2.5 \mathrm{hr}$, the reaction was stopped by the addition of $72 \mathrm{mg}$ of glycine. The solution was then chromatographed on a Sephadex G-150gel column $(2 \times 100 \mathrm{~cm})$, which was equilibrated with $0.1 \mathrm{M}$ potassium phosphate buffer ( $\mathrm{pH}$ 7.4) and calibrated with standard proteins (a high molecular weight kit $G$, Pharmacia). As a control experiment, UK was treated with glutaraldehyde under the same conditions in the absence of HSA fragments.

Preparation of antisera. Each solution of the unmodified UK (3.2 mg), HSA (20 mg), HSA fragments $(20 \mathrm{mg})$, and the conjugate of UK and HSA fragments $(3.2 \mathrm{mg})$ in $0.85 \% \mathrm{NaCl}$ was emulsified with an equal volume of Freund's complete adjuvant, and used in several portions for immunization of rabbits weighing about $2.5 \mathrm{~kg}$. Whole blood was drawn about 45 days after the initial immunization, was allowed to clot at $37^{\circ} \mathrm{C}$ for $1 \mathrm{hr}$, and was then kept at $4 \mathrm{C}$ overnight. Sera were separated from clots and stored at $-20^{\circ} \mathrm{C}$. Immunodiffusion analysis was done by the procedure of Ouchterlony. ${ }^{17)}$

Measurements of in vivo and in vitro stabilities. The in vitro inhibition of UK by protease inhibitors in sera was examined by measuring the remaining $U K$ activity after incubation with rat plasma. The enzyme solution $(40 \mu 1$, $13,500 \mathrm{IU} / \mathrm{ml}$ ) was incubated with $4 \mathrm{ml}$ of rat plasma at $37^{\circ} \mathrm{C}$, and $0.2-\mathrm{ml}$ samples of the reaction mixture were

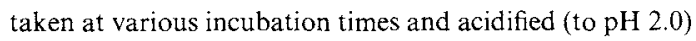
by the addition of $1 \mathrm{~N} \mathrm{HCl}$ to inactivate the remaining protease inhibitors in plasma ${ }^{18)}$ After standing for $30 \mathrm{~min}$, the solutions were neutralized (to $\mathrm{pH} 7.4$ ) with $1 \mathrm{~N} \mathrm{NaOH}$, and the remaining $\mathrm{UK}$ activity was measured.

The in vivo plasma clearance of UK and the conjugate with HSA fragments was studied by injecting the enzyme solution $(9,000 \mathrm{IU} / \mathrm{kg})$ into the femoral vein of Wistar rats weighing about $250 \mathrm{~g}$, which were anesthetized in advance by an intraperitoneal injection of $12.5 \mathrm{mg}$ of sodium pentobarbital. The blood samples were taken from the abdominal aorta at various times after the injection, and mixed with one tenth its volume of $3.8 \%$ sodium citrate solution (anti-coagulant). The supernatant solution obtained by brief centrifugation was acidified and neutralized as described above, and then was used for the assay of remaining $\mathrm{UK}$.

\section{Results}

\section{Fragmentation of $H S A$ and modification of $U K$ with $H S A$ fragments}

HSA is a natural polymer suitable as a chemical modifier of therapeutic enzymes because of its non-immunogenicity to humans. However, it has a large molecular weight 
$(67,000)$, and modification with it may exercise an inhibitory effect by steric hindrance on fibrinolytic enzymes such as UK. Therefore, we cleaved HSA with cyanogen bromide and then with agarose-immobilized trypsin into peptide fragments with molecular weights of less than 10,000 . Digestion with the immobilized trypsin was done for $4 \mathrm{hr}$, because the digestion for a longer period (e.g., $6 \mathrm{hr}$ ) under the same conditions resulted in formation of much smaller peptide fragments with molecular weights of less than 2000 , and that for a shorter period (e.g., $3 \mathrm{hr}$ ) was insufficient to produce fragments with molecular weights of less than 10,000 as judged from gel electrophoresis (data not shown). The cleaved product of HSA migrated as a broadly smearing band of stained protein by urea/SDS/ polyacrylamide gel electrophoresis (Fig. 1), showing that it is composed of several peptide fragments in a molecular weight range of $1,000 \sim 7,000$. The mixture of HSA fragments was then covalently attached to UK with glutaraldehyde as a coupling reagent. After the coupling reaction at $4^{\circ} \mathrm{C}$ for $2.5 \mathrm{hr}$, the remaining UK activity was $33 \%$ of the initial enzyme activity. The decrease in activity was probably due to the modification of amino groups of UK with glutaraldehyde, but not due to the steric hindrance derived from incorporation of the HSA fragments, since the remaining UK activity was about $23 \%$ when it was treated with glutaraldehyde in the absence of HSA fragments. To isolate the conjugate of $U K$ and HSA fragments produced, the reaction mixture was chromatographed on a Sephadex G150 gel column. As shown in Fig. 2, the conjugate was obtained as protein peaks eluted ahead of the unmodified UK (mol wt., 54,000) in fractions corresponding to molecular weights of $70,000 \sim 170,000$. The conjugate in the major peak (fraction No. $47 \sim 59$ ) had a specific UK activity of 57,400 IU per mg of protein, which is about $40 \%$ of the unmodified enzyme activity $(143,000 \mathrm{IU} / \mathrm{mg})$. On the basis of an averaged molecular weight of the conjugate $(70,000)$ as measured by SDS/ polyacrylamide gel electrophoresis (Fig. 1),

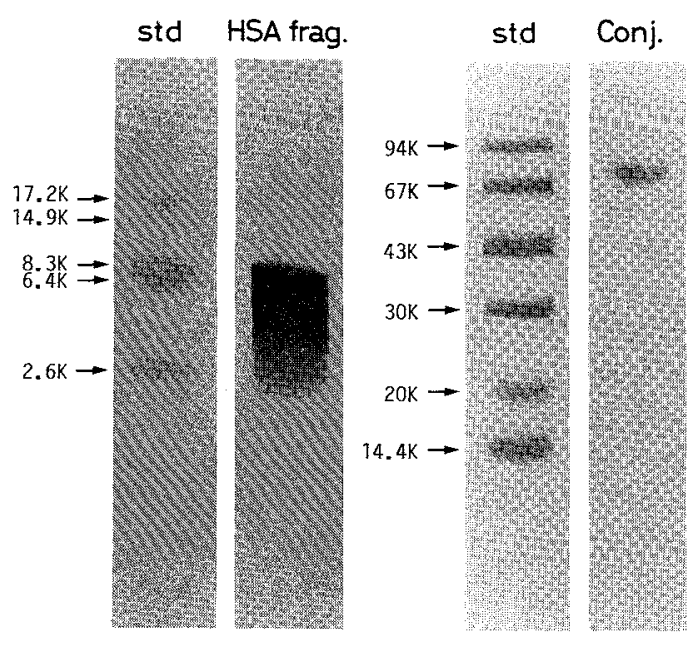

Fig. 1. Urea/SDS/Polyacrylamide Gel Electrophoresis of HSA Fragments (left) and SDS/Polyacrylamide Gel Electrophoresis of the Conjugate of UK and HSA Fragments (right).

The electrophoresis was done in $12 \%$ acrylamide gels for the HSA fragments and in $10 \%$ acrylamide gels for the conjugate. Sample load was $50 \mu \mathrm{g}$ (HSA fragments) and $10 \mu \mathrm{g}$ (the conjugate) per gel. Standard proteins were myoglobin and its fragments (left); and phosphorylase $b$, bovine serum albumin, egg albumin, carbonic anhydrase, trypsin inhibitor, and $\alpha$-lactalbumin (from top to bottom, right).

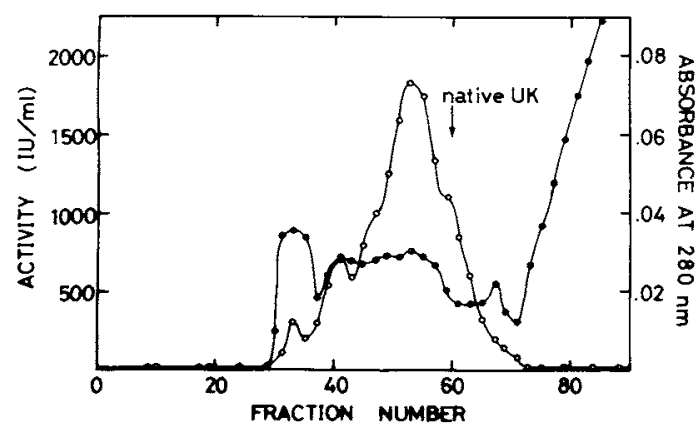

Fig. 2. Gel Filtration of UK Conjugated with HSA Fragments on Sephadex G-150.

The UK activity $(O)$ was measured on fibrin gel plates, and the protein concentration (O) was measured by absorbance at $280 \mathrm{~nm}$. Each fraction contained $3.2 \mathrm{ml}$.

at least $2 \sim 3$ molecules of HSA fragments were probably attached to one UK molecule, and the molecular activity corrected for the weight percentage of UK in the conjugate was estimated as about $53 \%$ of the unmodified enzyme activity. Since the minor prod- 
ucts eluted in fractions No. $30 \sim 46$ (Fig. 2) with molecular weights of $120,000 \sim 170,000$ had much lower specific activities $(22,300 \sim$ $6,100 \mathrm{IU} / \mathrm{mg}$ ), the conjugate with a molecular weight of 70,000 was used in the following experiments.

\section{Inactivation by protease inhibitors in rat plasma}

$\mathrm{UK}$ is a serine protease similar to trypsin and plasmin, and is known to be inactivated significantly by protease inhibitors present in sera. ${ }^{18)}$ We examined the inhibitory effects of rat plasma on the UK activity of the conjugate of UK and HSA fragments. As shown in Fig. 3 , the conjugate was more resistant to protease inhibitors than the unmodified UK; about 70 and $40 \%$ of the activity of the conjugate remained after incubation for 5 and $60 \mathrm{~min}$, respectively, with rat plasma, while the unmodified UK lost about 50 and $80 \%$ of its initial activity. Thus, the HSA fragments proteced UK, though partially, from inactivation by protease inhibitors in rat plasma, presumably because of steric hindrance in binding of the inhibitors with UK.

\section{In vivo stability during circulation in blood}

When the conjugate of UK and HSA fragments was injected into a femoral vein of Wistar rats, it showed a markedly high stability during circulation in blood (Fig. 4); its half-life of plasma clearance was about $8 \mathrm{~min}$ as an average of four measurements, which is ten times longer than that $(0.8 \mathrm{~min})$ of the unmodified enzyme. Thus, the in vivo stability of UK was significantly enhanced by modification with the HSA fragments.

\section{Immunological properties}

To examine the antigenicity (reactivity with an antibody) and the immunogenicity (elicitability of an antibody) of the synthesized conjugate by Ouchterlony double-immunodiffusion analyses, we obtained antibodies against UK, HSA, HSA fragments, and the conjugate of UK and HSA fragments from each of the immunized rabbits. In plate (1) of Fig. 5 where the anti-UK antiserum was added

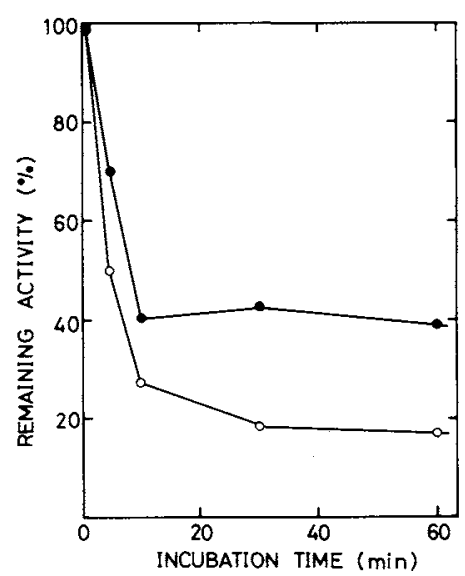

Fig. 3. Inactivation of UK by Protease Inhibitors in Rat Plasma.

The unmodified UK $(O)$ and the conjugate of UK and HSA fragments (O) were incubated with rat plasma at $37^{\circ} \mathrm{C}$. The remaining $\mathrm{UK}$ activity was measured at indicated times as described in the text.

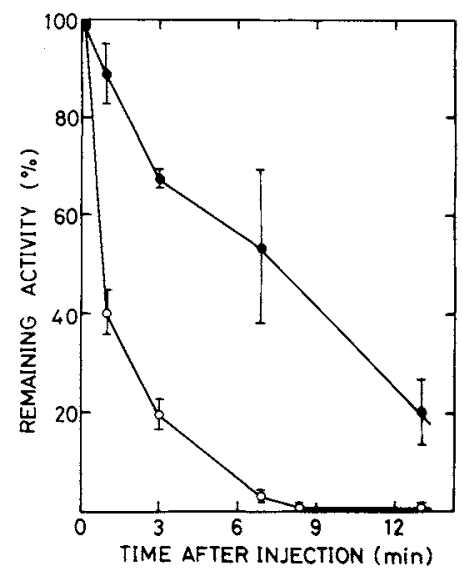

Fig. 4. Plasma Clearance of UK Activity after Intravenous Injection of the Unmodified UK and the Conjugate of UK and HSA Fragments

The unmodified UK $(O)$ and the conjugate $(O)$ were injected into four Wistar rats, and the remaining UK activity in blood samples taken at indicated times was measured as described in the text. Vertical bars show the standard error, and points are the means for four measurements.

in the center well, the antigenic proteins including UK, UK treated with glutaraldehyde, and the conjugate of mol wt. 70,000 reacted with the antiserum to form single precipitation lines, which fused with each other. However, 


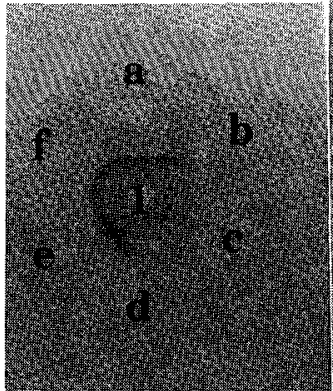

(1)

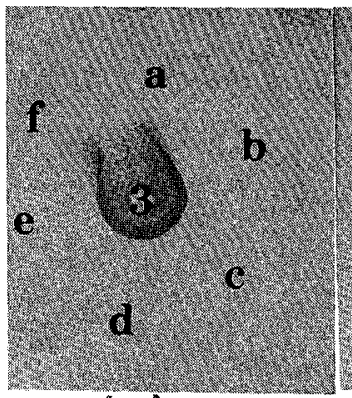

(3)

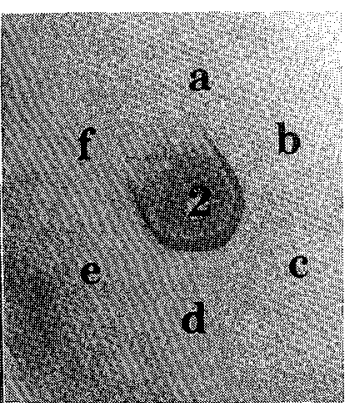

(2)

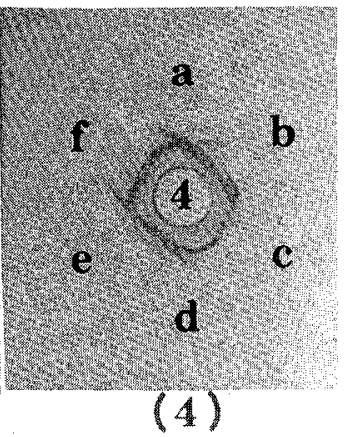

Fig. 5. Ouchterlony Immunodiffusion Analysis of the Conjugate of UK and HSA Fragments.

Immunodiffusion was done at $37^{\circ} \mathrm{C}$ for $16 \mathrm{hr}$. Center wells contained $10 \mu 1$ each solution of (1) anti-UK antiserum, (2) anti-HSA antiserum, (3) anti-HSA fragments antiserum, and (4) anti-conjugate antiserum. Peripheral wells contained (a) $10 \mu \mathrm{g}$ of $\mathrm{UK}$, (b) $10 \mu \mathrm{g}$ of HSA, (c) $100 \mu \mathrm{g}$ of HSA fragments, (d) $30 \mu \mathrm{g}$ of the conjugate with mol wt. 170,000 , (e) $20 \mu \mathrm{g}$ of the conjugate with mol wt. 70,000 , and (f) $10 \mu \mathrm{g}$ of UK treated with glutaraldehyde.

the conjugate with a molecular weight of about 170,000 (the number of HSA fragments attached to a UK molecule is estimated as 15 to 30 on the basis of its molecular weight) did not form a precipitation line. These results show that modification of UK with only a few HSA fragments does not affect the antigenicity of UK, but excessive modification with many fragments abolishes it, probably because all the epitopes on the UK molecule have been masked. In plate (2) where the anti-HSA antiserum was added in the center well, both the conjugates of mol wt. 70,000 and 170,000 as well as HSA fragments reacted with the antiserum, and their precipitation lines fused with that of HSA. Thus, the antigenicity of HSA was not lost by fragmentation into smaller peptides. The anti-HSA fragments antiserum in plate (3) also reacted with HSA, HSA fragments, and both the conjugates, suggesting that the immunogenicity of HSA fragments is identical with that of HSA. In plate (4), the precipitation line formed between the conjugate and the antiserum against it spurred with the lines formed in other proteins. In addition, the same antiserum reacted with both UK and HSA, the lines of which crossed each other. These results indicate that the conjugate of UK and HSA fragments possesses two distinct immunogenicities of UK and HSA, but it does not elicit new specific antibodies in the immunized rabbits.

\section{Discussion}

In these studies, we aimed to improve by chemical modification the very low stabilities of UK, particularly, its very short in vivo life, which is the most disadvantageous feature in the therapeutic use of UK. The rapid clearance of UK from the bloodstream is governed mainly by the liver, and is dependent on neither the active site structure of UK itself nor the presence of carbohydrate side chains on the surface of UK molecules. ${ }^{9)}$ Therefore, chemical modification at the active site or removal of the carbohydrate side chains would be ineffective for prolongation of the in vivo life of UK. Since the administered UK is cleared from blood predominantly by proteolytic digestion in the liver, covalent attachment of polymers that cover the cleavage sites on UK molecules is expected to give a longlived UK resistant to degradation by the liver. It might also provide UK with improved stability for inactivation by protease inhibitors present in blood.

Four kinds of UK inhibitors are known to occur in sera; $\alpha_{1}$-antitrypsin, ${ }^{19)} \alpha_{2}$-macroglobulin, ${ }^{20)}$ antithrombin III, ${ }^{20)}$ and plasminogen activator inhibitor. ${ }^{21)}$ The former three inhibit $\mathrm{UK}$ at a rather low reaction rate $(38 \sim 84 \%$ inactivation for $3 \sim 5 \mathrm{hr}$ ), while the last inactivates UK almost instantaneously. Our experiments of the UK inactivation by rat plas- 
ma (Fig. 3) demonstrate that the conjugate of UK and HSA fragments is insusceptible of long-term inhibition and also is partially resistant to the rapid inactivation within $10 \mathrm{~min}$. These results show the higher stability of the conjugate for inactivation by these serum protease inhibitors than the native UK. However, the in vivo stability of UK is more important for clinical application, because its half-life during circulation in blood is less than a minute due to the rapid degradation in the liver. ${ }^{9)}$ We found that the UK conjugated with HSA fragments was markedly stable upon injection into Wistar rats, the in vivo half-life being lengthened about ten times (Fig. 4). Therefore, the chemical modification of UK with HSA fragments also affects the clearance rate by the liver.

HSA fragments appear to be suitable as soluble polymers for the chemical modification of UK, because, as demonstrated in this paper, the immunological properties are identical with those of the native HSA, which is nonimmunogenic to humans. Mori et al. ${ }^{22)}$ and Miyaura et al. $^{23)}$ also reported that albumin fragments with molecular weights of around 10,000 formed in human urine show the same immunological properties as the native albumin. The chemically modified UK with HSA fragments gained the antigenicity of HSA, but no new antigenic determinants specific for the conjugate. Instead, it lost the antigenicity of UK when modified excessively with many HSA fragments. These results suggest the usefulness of HSA fragments for chemical modification of therapeutic enzymes.

A one-shot dosage of a large amount of UK leads to an immediate consumption of protease inhibitors in blood and also a rapid degradation of clotting factor by the activated plasmin, and thereby brings various side effects such as the tendency to hemorrhage due to the lowered activity in blood coagulation. ${ }^{24)}$ Although the conjugate of UK and HSA fragments showed only about $40 \%$ of the original UK activity, its considerable activity remained in sera of the injected rats even after disappearance of the activity of unmodified
UK (see Fig. 4). Thus, the chemically modified $\mathrm{UK}$ with high in vivo stability may be effectively applicable to the treatment of thromboembolic symptoms, producing no serious side effects when administered gradually in such a manner as an intravenous drip.

Acknowledgment. We thank Dr. M. Izumiya and Dr. T. Kobayashi, Wakamoto Pharmaceutical Co., Ltd., for their helpful discussions.

\section{References}

1) M. H. Remy and M. J. Poznansky, Lancet, 2, 68 (1978).

2) K. Miyata, Y. Nakagawa, M. Nakamura, T. Ito, K. Sugo, T. Fujita and K. Tomoda, Agric. Biol Chem., 52, 1575 (1988).

3) A. Abuchowski, T. van Es, N. C. Palczuk and F. F. Davis, J. Biol. Chem., 252, 3578 (1977).

4) M. Igarashi, J. Takatsuka, T. Shiba, S. Takeuchi, T. Kinoshita, T. Nakahara and K. Shimizu, Blood \& Vessel, 13, 378 (1982)

5) J. W. Fenton and M. J. Fasco, Thromb. Res., 4, 809 (1974).

6) T. Astrup and I. Sterndorff, Proc. Soc. Exptl. Biol. Med., 81, 675 (1952).

7) L. Lorand, in "Methods in Enzymology," Vol. XLV, ed. by L. Lorand, Academic Press Inc., New York, 1976, p. 31.

8) A. Klausner, Biotechnology, 1, 330 (1983).

9) D. Collen, F. De Cock and H. R. Lijnen, Thromb. Haemostas., 52, 24 (1984).

10) K. Yokoigawa, K. Tanizawa and K. Soda, Agric, Biol. Chem., 48, 1587 (1984).

11) J. Ploug and N. O. Kjeldgaard, Biochim. Biophys. Acta, 24, 278 (1957).

12) G. H. Barlow, in "Methods in Enzymology," Vol. XLV, ed. by L. Lorand, Academic Press Inc., New York, 1976, p. 239.

13) O. H. Lowry, N. J. Rosebrough, A. L. Farr and R. J. Randall, J. Biol. Chem., 193, 265 (1951).

14) R. H. McMenamy, H. M. Dintzis and F. Watson, $J$. Biol. Chem., 246, 4744 (1971).

15) R. Axen and S. Ernback, Eur. J. Biochem., 18, 351 (1971).

16) R. T. Swank and K. D. Munkres, Anal. Biochem., 39, 462 (1971).

17) O. Ouchterlony, Progr. Allergy, 5, 1 (1958).

18) T.-C. Wun, W.-D. Schleuning and E. Reich, J. Biol. Chem., 257, 3276 (1982).

19) I. Clemmensen and F. Christensen, Biochim. Biophys. Acta, 429, 591 (1976).

20) E. K. Waller, W.-D. Schleuning and E. Reich, Biochem. J., 215, 123 (1983). 
21) J. Chmielewska, M. Rånby and B. Wiman, Thrombo. Res., 31, 427 (1983).

22) M. Mori, Y. Hayashi and A. Kawamura, Seibutsu Butsuri Kagaku, 19, 349 (1975).
23) S. Miyaura, Y. Matsuda, K. Yamaguchi and $\mathrm{H}$. Moriya, Chem. Pharm. Bull., 29, 855 (1981).

24) D. Collen, J. M. Stassen, M. Blaber, M. Winkler and M. Verstraete, Thromb. Haemostas., 52, 27 (1984). 